\title{
Earlier HIV diagnosis - are mobile services the answer?
}

\author{
N van Schaik, K Kranzer, R Wood, L-G Bekker
}

Objectives. To assess the age and gender differences of clients accessing mobile HIV counselling and testing (HCT) compared with clients accessing facility-based testing, and to determine the difference in HIV prevalence and baseline CD4 counts.

Methods. This was a prospective observational cross-sectional study of 3 different HIV testing services in Cape Town. We compared data on age, sex, HIV status and CD4 counts collected between August and December 2008 from a mobile testing service (known as the Tutu Tester), a primary health care clinic, and a district hospital.

Results. A total of 3820 individuals were tested: 2499 at the mobile, 657 at the clinic, and 664 at the hospital. Age and sex distribution differed across services, with the mobile testing more men and older individuals. HIV prevalence was lowest at the mobile (5.9\%) compared with the clinic (18.0\%) and hospital (23.3\%). Of the HIV-infected individuals from the mobile service, $75 \%$ had a CD4 count higher than 350 cells/ $\mu$ l compared with $48 \%$ and $32 \%$ respectively at the clinic and hospital. Age- and sex-adjusted risk for HIV positivity was 3.5 and 4.9 times higher in the clinic-based and hospital-based services compared with the mobile service.

Conclusion. Mobile services are accessed by a different population compared with facility-based services. Mobile service clients were more likely to be male and less likely to be HIV positive, and those infected presented with earlier disease.

S Afr Med J 2010; 100: 671-674.
HIV counselling and testing (HCT) services are important entry points for prevention and care. ${ }^{1}$ The efficacy and cost-effectiveness of traditional facility-based HCT have been well demonstrated in resource-limited settings. ${ }^{2-5}$

However, in the southern African countries with severe HIV epidemics, testing remains inadequate. Only $2-20 \%$ of individuals reported testing for HIV within the past 12 months in $2007 .^{6}$

Early diagnosis of HIV infection, through counselling and testing on a large scale, is critical to providing HIV-positive individuals with antiretroviral treatment (ART). ${ }^{1}$ In an attempt to reduce morbidity and mortality, new World Health Organization (WHO) guidelines recommend initiating ART at CD4 counts $<350$ cells $/ \mu 1 .{ }^{7.8}$ Conventional provider-initiated, health care facility-based services alone may not have the capacity to cater for increased demands. In addition, these stationary services may not appeal to most of the population, owing to issues of accessibility, acceptability and confidentiality. ${ }^{9-12}$ Mobile HCT services overcome some of these shortcomings ${ }^{9}$ but little is known about the differences between individuals accessing mobile HCT services compared with those tested at health care facilities.

We aimed to assess the age and gender differences of clients accessing mobile HCT compared with facility-based testing, and to determine the difference in HIV prevalence and baseline CD4 counts.

The Desmond Tutu HIV Centre, Institute for Infectious Disease and Molecular Medicine and Department of Medicine, Faculty of Health Sciences, University of Cape Town

N van Schaik, MB ChB

K Kranzer, MB BS

R Wood, MB BCh, BSc, FCP

L-G Bekker, MB ChB, FCP, PhD

Clinical Research Unit, Department of Infectious and Tropical Diseases, London School of Hygiene and Tropical Medicine, London

K Kranzer, MB BS

\section{Methods}

We compared data from 3 HIV testing services in Cape Town: mobile HIV testing was provided by the Tutu Tester, and facility-based data were collected from the HCT registers at a primary health care clinic and a district level hospital. Data on age, sex, HIV status and CD4 counts of clients over the age of 15 were collected between August and December 2008. HIV-positive individuals from the Tutu Tester who already knew their status were excluded from the analysis, as were antenatal HIV data.

Rapid HIV testing and subsequent CD4 count testing were performed according to the Provincial Government of the Western Cape's HIV testing guidelines. At the Tutu Tester, CD4 counts were initially made by point-of-care testing (POCT), but clients were subsequently offered both POCT and laboratory CD4 counts. To ensure comparability across services, only laboratory CD4 counts were used in the analysis.

\section{The Tutu Tester}

The Tutu Tester (Fig. 1) is a nurse-run, counsellor-supported mobile screening clinic run by the Desmond Tutu HIV Foundation. It operates 5 days a week mainly in underserviced peri-urban areas

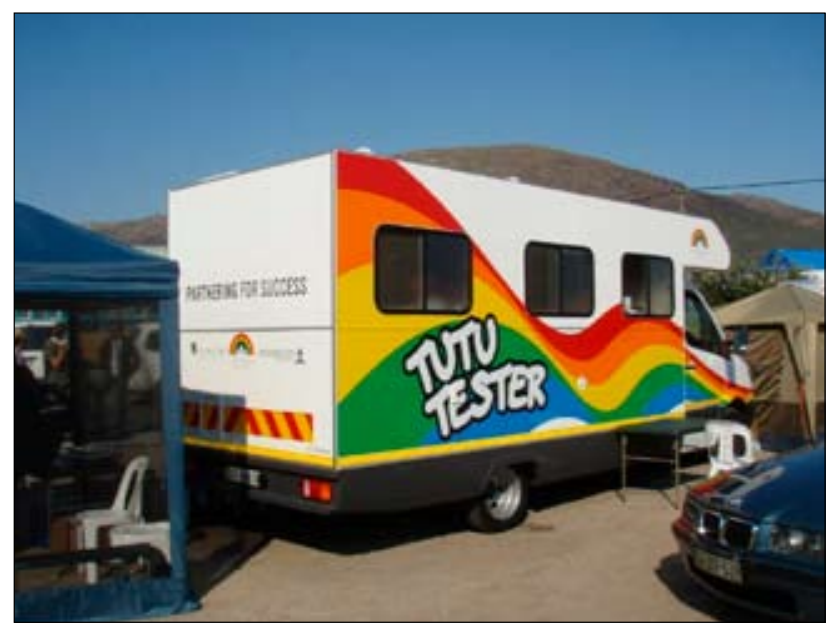

Fig. 1. The Tutu Tester, a mobile HIV testing and counselling service, in action in Cape Town. 
in greater Cape Town, and rotates through sites such as township shopping centres, taxi ranks and stations, and on the road side. As this service is not formally advertised, it attracts ambulatory clients who spontaneously access HIV testing. This client-initiated testing is offered in combination with screening for other chronic conditions such as hypertension, diabetes and obesity, in an attempt to normalise testing.

\section{Community clinic}

This primary health care clinic is in a peri-urban settlement in the southern sub-district of Cape Town. The clinic provides HCT, ART, child care, immunisations, family planning and services for tuberculosis (TB) and sexually transmitted infections (STI). Adults with other acute or chronic medical conditions are referred to secondary level hospitals. HCT is largely provider-initiated through TB and STI services, but some clients attend specifically for HCT.

\section{District hospital}

This district hospital services the peri-urban settlement mentioned above as well as the surrounding communities. Most patients testing at the hospital have a medical indication for testing, although some are self-referrals.

Statistical analysis was performed using STATA (Version 10.0, College Station, Texas). Proportions were calculated and stratified according to services, and chi-square testing was used to investigate significant differences in proportions. A logistic regression model was used to calculate HIV risk in the different services, adjusted for age and sex.
Ethical permission for the study was provided by the Ethics Committee of the University of Cape Town.

\section{Results}

A total of 3820 individuals were tested for HIV between August and December 2008 (Table I). The overall HIV prevalence was 11.0\%. The prevalence for clients tested at the mobile HCT service was $5.9 \%$, for clients tested at the primary health care clinic $18.0 \%$, and the district hospital $23.3 \%$.

The mobile service tested a significantly higher proportion of men (51.5\%) compared with the stationary services $(40.2 \%$ and $39.6 \%$ respectively). The age distribution was significantly different across the services, with a higher proportion of $>35$-year-olds tested at the mobile service.

Laboratory CD4 results were available for $44.2 \%(65 / 147)$ of the HIV-positive individuals at the mobile service, 65.3\% (77/118) at the primary health care clinic, and $42.6 \%(66 / 155)$ at the hospital. More than $75 \%$ of the HIV-infected individuals at the mobile service had a CD4 count $>350$ cells/ $\mu$ l, compared with $48.1 \%$ and $32.0 \%$ at the clinic and hospital respectively. No HIV-positive individual was found to have a CD4 count $<100$ cells/ $\mu$ at the mobile service, compared with $11.7 \%$ at the clinic and $34.0 \%$ at the hospital.

Univariate analysis showed that the risk of testing HIV positive was 3.5 (95\% confidence interval (CI) 2.7 - 4.5) and 4.9 (95\% CI 3.8 - 6.2) times higher in clinic-based and hospital-based services than the mobile service (Table II). Women were 1.5 (95\% CI 1.2 - 1.8) times more likely to test HIV positive than men. The risk for testing HIV positive was highest among individuals aged 31 - 35. Multivariate analysis showed similar results.

Table I. Analysis by site of HIV result, gender, age and baseline CD4 count

\begin{tabular}{|c|c|c|c|c|c|c|c|c|c|}
\hline \multirow[b]{2}{*}{ Variables } & \multicolumn{2}{|c|}{$\begin{array}{c}\text { Total } \\
(N=3820)\end{array}$} & \multicolumn{2}{|c|}{$\begin{array}{l}\text { Mobile VCT } \\
\qquad(N=2499)\end{array}$} & \multicolumn{2}{|c|}{$\begin{array}{l}\text { Clinic-based VCT } \\
\qquad(N=657)\end{array}$} & \multicolumn{2}{|c|}{$\begin{array}{l}\text { Hospital-based VCT } \\
\qquad(N=664)\end{array}$} & \multirow[b]{2}{*}{$p$-value } \\
\hline & $N$ & $\%$ & $N$ & $\%$ & $N$ & $\%$ & $N$ & $\%$ & \\
\hline \multicolumn{10}{|l|}{ HIV } \\
\hline Negative & 3400 & 89.0 & 2352 & 94.1 & 539 & 82.0 & 509 & 76.7 & $<0.01$ \\
\hline Positive & 420 & 11.0 & 147 & 5.9 & 118 & 18.0 & 155 & 23.3 & \\
\hline \multicolumn{10}{|l|}{ Gender } \\
\hline Male & 1812 & 47.5 & 1285 & 51.5 & 264 & 40.2 & 263 & 39.6 & $<0.01$ \\
\hline Female & 2003 & 52.5 & 1209 & 48.5 & 393 & 59.8 & 401 & 60.4 & \\
\hline \multicolumn{10}{|l|}{ Age (years) } \\
\hline$<26$ & 1436 & 37.7 & 843 & 33.8 & 335 & 51.0 & 258 & 39.5 & $<0.01$ \\
\hline $26-30$ & 676 & 17.8 & 391 & 15.7 & 148 & 22.5 & 137 & 21.0 & \\
\hline $31-35$ & 477 & 12.5 & 299 & 12.0 & 79 & 12.0 & 99 & 15.1 & \\
\hline $36-40$ & 329 & 8.6 & 234 & 9.4 & 37 & 5.6 & 58 & 8.9 & \\
\hline $41-50$ & 495 & 13.0 & 390 & 15.6 & 40 & 6.1 & 65 & 9.9 & \\
\hline$>50$ & 393 & 10.3 & 338 & 13.6 & 18 & 2.7 & 37 & 5.7 & \\
\hline \multicolumn{10}{|c|}{ CD4 (cells/ $\mu \mathrm{l})$} \\
\hline$<100$ & 26 & 13.5 & 0 & 0.0 & 9 & 11.7 & 17 & 34.0 & $<0.01$ \\
\hline $100-200$ & 23 & 12.0 & 7 & 10.8 & 9 & 11.7 & 7 & 14.0 & \\
\hline $201-350$ & 41 & 21.4 & 9 & 13.9 & 22 & 28.6 & 10 & 20.0 & \\
\hline $351-500$ & 38 & 19.8 & 16 & 24.6 & 18 & 23.4 & 4 & 8.0 & \\
\hline$>500$ & 64 & 33.3 & 33 & 50.8 & 19 & 24.7 & 12 & 24.0 & \\
\hline
\end{tabular}


Table II. HIV risk: Univariate and multivariate analyses

\begin{tabular}{|c|c|c|c|c|}
\hline \multirow[b]{2}{*}{ Variables } & \multicolumn{2}{|c|}{ Univariate analysis } & \multicolumn{2}{|c|}{ Multivariate analysis } \\
\hline & OR & $95 \%$ CI & OR & $95 \%$ CI \\
\hline \multicolumn{5}{|l|}{ Site } \\
\hline Mobile VCT & 1 & & & \\
\hline Clinic-based VCT & 3.50 & $2.70-4.54$ & 3.70 & $2.82-4.85$ \\
\hline Hospital-based VCT & 4.87 & $3.81-6.22$ & 4.77 & $3.70-6.14$ \\
\hline \multicolumn{5}{|l|}{ Gender } \\
\hline Male & 1 & & & \\
\hline Female & 1.49 & $1.21-1.83$ & 1.42 & $1.14-1.76$ \\
\hline \multicolumn{5}{|l|}{ Age (years) } \\
\hline$<26$ & 1.00 & & & \\
\hline $26-30$ & 2.02 & $1.52-2.70$ & 2.15 & $1.60-2.90$ \\
\hline $31-35$ & 2.59 & $1.91-3.51$ & 3.04 & $2.21-4.18$ \\
\hline $36-40$ & 2.19 & $1.53-3.13$ & 2.85 & $1.96-4.14$ \\
\hline $41-50$ & 1.22 & $0.85-1.75$ & 1.76 & $1.21-2.57$ \\
\hline$>50$ & 0.88 & $0.57-1.36$ & 1.44 & $0.92-2.27$ \\
\hline $\mathrm{OR}=$ odds ratio. & & & & \\
\hline
\end{tabular}

\section{Discussion}

Our study shows that the mobile HCT service is accessed by a very different population with regard to age, sex, HIV prevalence and immune deficiency, and this despite the facility-based services being in areas in which the mobile testing unit operates.

HIV prevalence for the Cape Metropolitan area was estimated to be $18.3 \%$ in 2008 . The Tutu Tester operates in predominantly underserviced, high-prevalence areas. The prevalence in Khayelitsha was $33.4 \%$ and in Klipfontein $23.4 \%$ in $2008 .{ }^{13}$ HIV prevalence in adults in the mentioned peri-urban settlement was estimated to be $25 \%$ in $2008 .{ }^{14}$

Most of the HIV-positive clients tested at the mobile service had a CD4 count $>350$ cells $/ \mu$, indicating that the mobile service facilitates earlier diagnosis. This is particularly important in view of the latest WHO guidelines recommending initiation of ART at a CD4 threshold of 350 cells/ $\mu$.

Mobile testing appears to be more acceptable to men, as significantly more men were tested at the mobile service. This is important, as the 2008 South African National Survey has again shown that more females test for HIV than males. ${ }^{15}$ The convenience and accessibility of the mobile service compared with stationary services may account for the difference. ${ }^{9,10,12}$ Combining HIV testing with screening for other chronic conditions may reduce stigma, hence encouraging testing in a wider population including males and the elderly.

This study has several limitations. A substantial number of CD4 counts were missing owing to POCT results being excluded from the analysis and CD4 counts, if done, not being recorded in the facility HCT registers. These data were probably missing at random and are therefore unlikely to introduce a significant bias. Known HIV-positive individuals who retested for HIV at the mobile service were excluded from the analysis. Data on prior testing were not available for facility-based services and it was therefore assumed that individuals testing positive at the clinic or hospital were newly diagnosed with HIV. This might have led to an overestimation of the HIV prevalence in the stationary services. However, HIV prevalence in the mobile population was $10.2 \%$ when including re-testers, which is still much lower than the HIV prevalence at the clinic and hospital. This operational research study also compares data from only 3 services, which may not be representative of mobile and stationary services generally. However, this study shows that mobile HIV testing services, which have not been widely used in South Africa, can offer HIV testing to a healthier community, which will be important if treatment guidelines are altered in South Africa.

HIV testing and knowledge of HIV status are the first steps in linking individuals to HIV care and ART. For ART benefits to be fully realised, linkage to care, timely treatment initiation, adherence to ART, and retention in care are critical. Linkage to care is particularly important in a mobile setting, as these services are not able to deliver treatment and must refer patients to stationary facilities. The costeffectiveness of mobile testing also needs to be analysed.

In summary: mobile services are accessed by a different population than facility-based services. Mobile service clients were more likely to be male and less likely to be HIV positive, and those infected presented with earlier disease.

We thank the dedicated team of individuals who work daily on the Tutu Tester and have tested thousands of individuals; Dr Rob Martell, Superintendent at False Bay Hospital and his staff; and Sr Traut (Masiphumelele Clinic Facility Manager) and her staff.

Funding. The staff, medical supplies and running costs were made possible by funding through the Anova Health Institute and the United States Agency for International Development (USAID) through PEPFAR. Further funding was given by the Higher Education HIV/AIDS Programme (HEAIDS) and its partners - the Department of Education, the European Union and Higher Education South Africa. The Tutu Tester vehicle and CD4 machine were sponsored by Metropolitan Health Group and QUALSA.

\section{References}

1. UNAIDSWHO Policy Statement on HIV Testing. Geneva: UNAIDS/WHO, 2004. http://www.who.int/hiv/pub/vct/statement/en/ (accessed 5 December 2009).

2. Weinhardt LS, Carey MP, Johnson BT, Bickham NL. Effects of HIV counseling and testing on sexual risk behavior: a meta-analytic review of published research, 1985-1997. Am J Public Health 1999;89(9):1397-1405.

3. Thielman NM, Chu HY, Ostermann J, et al. Cost-effectiveness of free HIV voluntary counseling and testing through a community-based AIDS service organization in Northern Tanzania. Am J Public Health 2006;96(1):114-119. 
4. Sweat M, Gregorich S, Sangiwa G, et al. Cost-effectiveness of voluntary HIV-1 counselling and testing in reducing sexual transmission of HIV-1 in Kenya and Tanzania. Lancet 2000; 356(9224):113-121.

5. The Voluntary HIV-1 Counseling and Testing Efficacy Study Group. Efficacy of voluntary HIV-1 counselling and testing in individuals and couples in Kenya, Tanzania, and Trinidad: a randomised trial. Lancet 2000;356(9224):103-112.

6. Peltzer K, Matseke G, Mzolo T, Majaja M. Determinants of knowledge of HIV status in South Africa: results from a population-based HIV survey. BMC Public Health 2009;9(174):174.

7. WHO (2009) RAPID ADVICE - Antiretroviral Therapy for HIV Infection in Adults and Adolescents. Geneva: World Health Organization, 2009. http://www. who.int/hiv/pub/arv/advice/en/index.html (accessed 5 December 2009).

8. Sterne JA, May M, Costagliola D, et al. Timing of initiation of antiretroviral therapy in AIDS-free HIV-1-infected patients: a collaborative analysis of $18 \mathrm{HIV}$ cohort studies. Lancet 2009; 373(9672):1352-1363.

9. Morin SF, Khumalo-Sakutukwa G, Charlebois ED, et al. Removing barriers to knowing HIV status: same-day mobile HIV testing in Zimbabwe. J Acquir Immune Defic Syndr 2006;41(2):218-224.

10. Matovu JK, Makumbi FE. Expanding access to voluntary HIV counselling and testing in sub-Saharan Africa: alternative approaches for improving uptake, 2001-2007. Trop Med Int Health 2007;12(11):1315-1322.
11. Hutchinson PL, Mahlalela X. Utilization of voluntary counseling and testing services in the Eastern Cape, South Africa. AIDS Care 2006;18(5):446-455.

12. Angotti N, Bula A, Gaydosh L, Kimchi EZ, Thornton RL, Yeatman SE. Increasing the acceptability of HIV counseling and testing with three C's: convenience, confidentiality and credibility. Soc Sci Med 2009;68(12):2263-2270.

13. HIV and Syphilis Prevalence in the Western Cape: Results of the 2008 HIV \& Syphilis Antenatal Provincial and Sub district Surveys. Cape Town: Provincial Government Western Cape, Department of Health, 2008.

14. Middelkoop K, Wood R, Myer L, et al. Widespread ART is associated with decline in TB prevalence. 5th IAS Conference on HIV Pathogenesis, Treatment and Prevention. Cape Town, 2009.

15. Shisana O, Rehle T, Simbayi L, et al. South African National HIV prevalence, incidence, behaviour and communication survey 2008: A turning tide among teenagers? Cape Town: HSRC, 2009. http://www.hsrcpress.ac.za/product.php?p roductid $=2264 \&$ cat $=1$ \&page $=3 \&$ freedownload $=1$ (accessed 5 December 2009).

Accepted 25 June 2010. 